\title{
Is cerebral palsy a cause of death?
}

\author{
Jennifer M Ryan ${ }^{1,2}$ iD, Nicola Ryan ${ }^{3,4}$, \\ Mark D Peterson ${ }^{5}$ iD \\ 1 Department of Public Health and Epidemiology, Royal College of Surgeons in \\ Ireland, Dublin, Ireland. 2 Institute of Environment, Health and Societies, Brunel \\ University London, London; 3 Department of Cardiology, Aberdeen Royal \\ Infirmary, Aberdeen, UK. 4 Department of Interventional Cardiology, Hospital \\ Clínico San Carlos, Madrid, Spain. 5 Department of Physical Medicine and \\ Rehabilitation, University of Michigan Medicine, Ann Arbor, MI, USA.
}

Correspondence to: jenniferryan@rcsi.com

doi: $10.1111 /$ dmen. 14440

EDITOR-The commentary on our paper ${ }^{1,2}$ prompted us to further explore our data on coding cerebral palsy (CP) as the underlying cause of death. The commentary notes that 'it would prove informative to publish the specific cause of death...for the 142 decedents with CP'. Unfortunately, we are unable to report many causes of death because data restrictions prevent us from reporting the cause of death where five or less deaths are observed. However, we can report that 11 deaths $(7.7 \%)$ in our study were due to diseases of the nervous system that did not include CP (i.e. International Classification of Diseases, 10th Revision [ICD-10] codes G00-G99, not including G80.0-G80.9) and 11 deaths (7.7\%) were due to diseases of the digestive system (ICD-10 codes K00-K93). This number remained the same regardless of revising the cause of death where the underlying cause of death was coded as CP.

The second point relates to recoding cause of death where the underlying cause was coded as CP. We first provide more detail on how cause of death is recorded and tabulated in England.

A medical practitioner completes the Medical Certificate of Cause of Death (MCCD) when the death is certified. Causes of death that are entered on the MCCD are defined as 'all those diseases, morbid conditions or injuries which either resulted in or contributed to death and the circumstances of the accident or violence which produced any such injuries'. When more than one cause of death is recorded, the underlying cause of death is selected for tabulation. Underlying cause of death is defined as '(a) the disease or injury which initiated the train of morbid events leading directly to death, or (b) the circumstances of the accident or violence which produced the fatal injury'. When completing the MCCD, the medical practitioner is asked to state the disease or condition leading directly to death on the first line and all morbid conditions, if any, giving rise to this cause on the lines below, with the underlying condition stated on the lowest line. ${ }^{3}$ The 'general principle' for selecting the underlying cause of death when more than one condition is entered is to 'select the condition entered alone on the lowest line, if it could have given rise to all the conditions entered above it'. ${ }^{3}$ If the general principle does not apply, a number of selection rules are used to select the underlying cause of death. ${ }^{3}$

In the data set used in our paper, several causes of death may be listed for each patient with an underlying cause of death specified. The order in which causes of death are listed does not necessarily correspond to the order they are listed on the MCCD. The underlying cause of death was CP for 37 patients (26\%). We only replaced CP as the underlying cause of death when just one additional cause of death was listed. In all cases, this additional cause of death preceded CP (i.e. it was listed as cause 1 when CP was cause 2). Therefore, for these patients, we replaced the underlying cause of death with the cause of death preceding CP. ${ }^{2}$ For the purpose of this letter, we provide standardized mortality ratios (SMRs) for malignant neoplasms, diseases of the respiratory system, and diseases of the circulatory system, when the underlying cause of death is not reattributed to another cause (Table $\mathrm{S} 1$, online supporting information). The result of this is that the number of deaths due to respiratory disease decreases. However, the cause-specific SMR is highest for diseases of the respiratory system, regardless of revising the underlying cause of death.

Ours is not the first study to demonstrate that coding cause of death as 'CP' has implications on the estimation of mortality due to respiratory disease. Strauss et al. ${ }^{4}$ found that $15 \%$ of deaths among people with $\mathrm{CP}(\geq 14 \mathrm{y})$ were due to respiratory disease. However, an additional 30\% had potentially uninformative codes such as CP and congenital anomalies. Without recoding these uninformative codes, Strauss et al. ${ }^{4}$ found that diseases of the circulatory system were the most common cause of death among adults with CP. Landes et al. ${ }^{5}$ recently examined the effect of revising the underlying cause of death, where the underlying cause was coded as a developmental disability, using a sequential underlying cause of death revision process. The underlying cause of death was coded as a developmental disability for $59.53 \%$ of adults with CP. Revision of the underlying cause of death resulted in the percentage of deaths due to diseases of the respiratory system increasing from $8.95 \%$ to $29 \%$. Diseases of the respiratory system rose from the third to the first leading cause of death among adults with $\mathrm{CP}$ as a result. Diseases of the circulatory system remained the second leading cause of death, regardless of revision.

The purpose of the previously stated definition of the underlying cause of death is to identify the precipitating cause of death in order to appropriately target public health interventions towards chronic debilitating diseases. ${ }^{3}$ 
However, as guidance for doctors completing MCCDs in England and Wales states, 'Doctors are expected to state the cause of death to the best of their knowledge and belief; they are not expected to be infallible' (https://assets. publishing.service.gov.uk/government/uploads/system/ uploads/attachment_data/file/757010/guidance-for-doc tors-completing-medical-certificates-of-cause-of-dea th.pdf).

According to the definition, it is plausible that $\mathrm{CP}$ is correctly coded as the underlying cause of death for some adults with CP. However, further discussion is needed about the usefulness of coding the underlying cause of death as $\mathrm{CP}$ from an epidemiological perspective, and the possibility of allowing $\mathrm{CP}$ to be superseded by one or more suitable causes when determining the underlying cause of death for tabulation.

\section{SUPPORTING INFORMATION}

The following additional material may be found online:

Table S1: Observed number of deaths and standardized mortality ratio for each cause for analyses where cause of death due to cerebral palsy was recoded and cause of death due to cerebral palsy was not recoded.

\section{REFERENCES}

1. Landes SD. Mortality risk among adults with cerebral palsy: alarming findings and lingering questions. Dev Med Child Neurol 2019; 61: 860-1.

2. Ryan JM, Peterson MD, Ryan N, et al. Mortality due to cardiovascular disease, respiratory disease, and cancer in adults with cerebral palsy. Dev Med Child Neurol 2019; 61: $924-8$.
3. World Health Organization. International Statistical Classification of Diseases and Related Health Problems, 10th Revision (ICD-10), Volume 2. Available at: https:// www.who.int/classifications/icd/ICD-10_2nd_ed_volume 2.pdf (accessed 15th November 2019).

4. Strauss D, Cable W, Shavelle R. Causes of excess mortality in cerebral palsy. Dev Med Child Neurol 1999; 41: 580-5.
5. Landes SD, Stevens JD, Turk MA. Obscuring the effect of coding developmental disability as the underlying cause of death on mortality trends for adults with developmental disability: a cross-sectional study using US Mortality Data from 2012 to 2016. BMF Open 2019; 9: e026614.

\section{Mac Keith Press Clinics in Developmental Medicine}

\section{Rett Syndrome}

Edited by Walter E Kaufmann

with Alan Percy, Angus Clarke, Helen Leonard and SakkuBai Naidu

- Provides a wide-ranging review of the clinical issues in Rett syndrome.

- Topics covered include clinical gentics, motor abnormalities, sleep issues and neurobiology.

- Approaches the disorder from initial examination to in-depth study.

- Discusses possible future treatments for the syndrome.

Contact us at admin@mackeith.co.uk to receive full table of contents and further details. Sept 2017 / 240 x 172mm / 240 pp / Hardback / ISBN 978-1-909962-83-5 / £70.00

www.mackeith.co.uk/shop 